\title{
Comparison of the accuracy of steroid placement with clinical outcome in patients with shoulder symptoms
}

\author{
J A Eustace, D P Brophy, R P Gibney, B Bresnihan, O FitzGerald
}

\begin{abstract}
Objective-To study the effect of accuracy on the clinical outcome of local steroid injections to the shoulder.

Methods-37 patients with shoulder symptoms of at least two months' duration received local injections of a mixture of triamcinolone and radiographic contrast material using a standardised technique. Radiographs of the joint were taken immediately afterwards. Details of the patients' symptoms (assessed by visual analogue scales) and range of movement at the joint were obtained before and two weeks after the injection. At follow up the patients were also assessed by means of a five point global rating scale of maximum and current benefit.
\end{abstract}

Results-14 of the 38 procedures (37\%) were judged to be accurately placed: four of the 14 attempted subacromial injections (29\%) and 10 of the 24 attempted glenohumeral injections $(42 \%)$. There were significant differences in relation to outcome between the accurately placed and the inaccurately placed groups.

Conclusions-Accuracy of steroid placement by injection in patients with shoulder symptoms may significantly affect the clinical outcome.

(Ann Rheum Dis 1997;56:59-63)

The local injection of steroid, either intra-articular or into periarticular structures such as bursae, has been used for many years to help relieve localised rheumatic symptoms. The procedure is cheap and when properly performed it is usually well tolerated. ${ }^{12}$ Provided it is not repeated too often at the same site it is generally accepted that it has a low incidence of side effects. ${ }^{3-5}$ While it continues to be widely used in everyday clinical practice, the studies which have examined the effectiveness of local steroid placement have had widely varying results. ${ }^{6-14}$ Part of this variability may relate to differences in the frequency with which the steroid is successfully injected into the desired target. The purpose of this study was to examine the effect that accuracy of steroid placement has on the outcome of local steroid injections.

\section{Methods}

Patients between the ages of 18 and 80 years with symptoms believed to be amenable to a localised steroid injection into either the glenohumeral joint or the subacromial bursa were considered eligible for the study. Symptoms had to be present for at least two months. For four weeks before injection the patients were prohibited from starting any new medications or from receiving any other local steroid injections. Significant symptoms had to be limited to the shoulder joint only; those subjects with background rheumatological complaints were excluded unless their underlying condition was at a stable baseline.

Patients with a history of allergy to steroids or to contrast material were excluded. The study protocol was approved by the hospital ethics committee and informed consent was obtained from each patient.

At study entry the details of any background rheumatological diagnosis, all current medications, and the nature and duration of the current shoulder problem were obtained. Symptoms, in the form of localised pain, stiffness, and loss of function, were measured by the patient marking a $10 \mathrm{~cm}$ visual analogue scale of between 0 and 10 . The passive range of movement of the affected joint was assessed clinically for flexion, abduction, and internal and external rotation. A goniometer was not used.

A standard technique for injection was used as outlined by Dixon. ${ }^{15}$ For glenohumeral injections an anterior approach was used, inserting a $21 \mathrm{G} \times 1.5$ inch needle $2.5 \mathrm{~cm}$ (1 in) below the tip of the coracoid process and 2.5 $\mathrm{cm}$ medial to the head of the humerus. Access to the subacromial bursa was gained from a lateral approach, passing the needle through the deltoid muscle and directing it medially and slightly anterior under the lateral end of the acromion process.

All patients with a diagnosis of adhesive capsulitis or acute synovitis of the shoulder joint were treated with a glenohumeral injection. Patients with rotator cuff syndrome received an injection into their subacromial bursa unless the point of maximum tenderness was located over the anterior glenohumeral joint line.

Both sites were injected using a combination of $1.5 \mathrm{ml}(15 \mathrm{mg})$ of triamcinolone mixed thoroughly with $3.5 \mathrm{ml}$ of non-ionic water soluble contrast material, Omnipaque $(300 \mathrm{mg}$ $\mathrm{I} \mathrm{ml}^{-1}$; Nycomed). To minimise dispersion after injection the subjects were injected in the radiology department and radiographs taken immediately afterwards. Two views were taken of each injected joint, a standard anteroposterior view and an axial view. The radiographs were interpreted by consensus, by two radiologists, both experienced in the interpretation of shoulder arthrograms. The figure shows an example of an accurately and an inaccurately injected glenohumeral joint. 
Following injection patients were asked to rest the shoulder for the remainder of the day and to continue with their usual medications until the follow up assessment. They were prohibited from receiving physiotherapy during this time. No restrictions were placed on the patients' ability to work or to use their shoulders as tolerated.

The subjects were reviewed two weeks after their injection with an assessment similar to that at study entry. The same doctor performed the baseline and follow up assessments for each patient. Neither the doctor nor the patient was aware of the
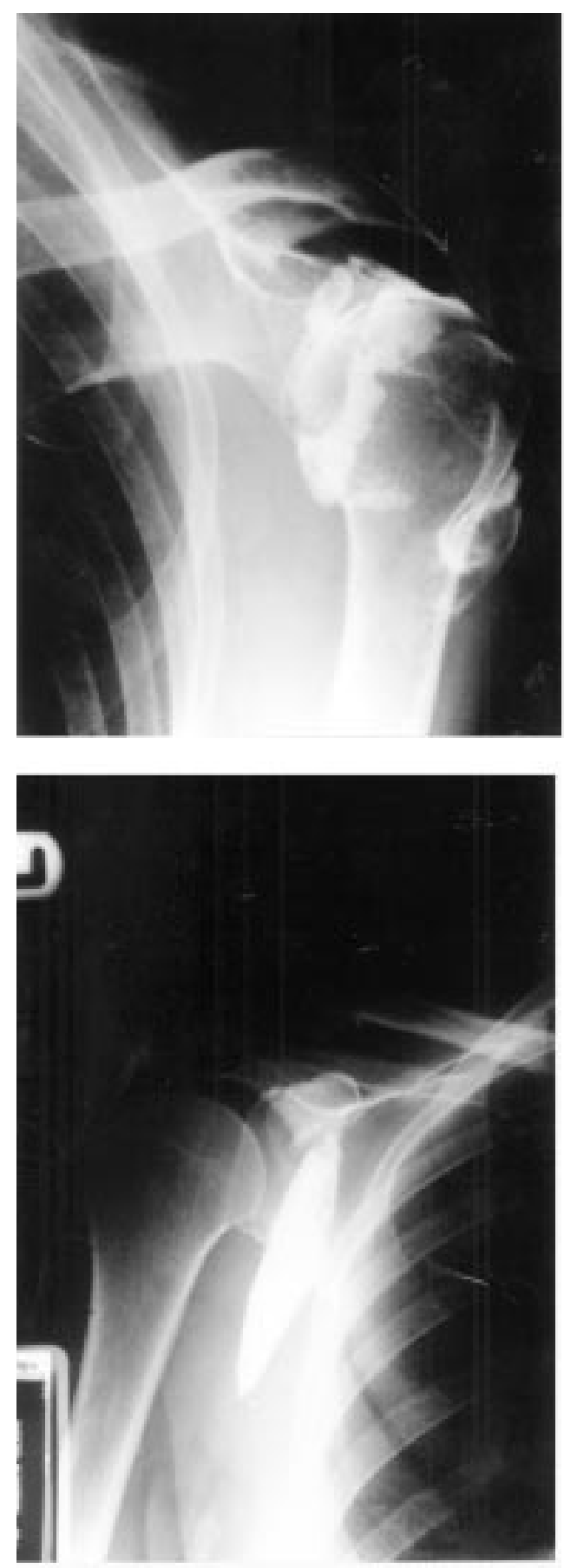

Sample antero-posterior and axial radiographs of an accurately injected (top) and inaccurately injected (bottom) glenohumeral joint, taken immediately after injection of steroid/contrast mixture.
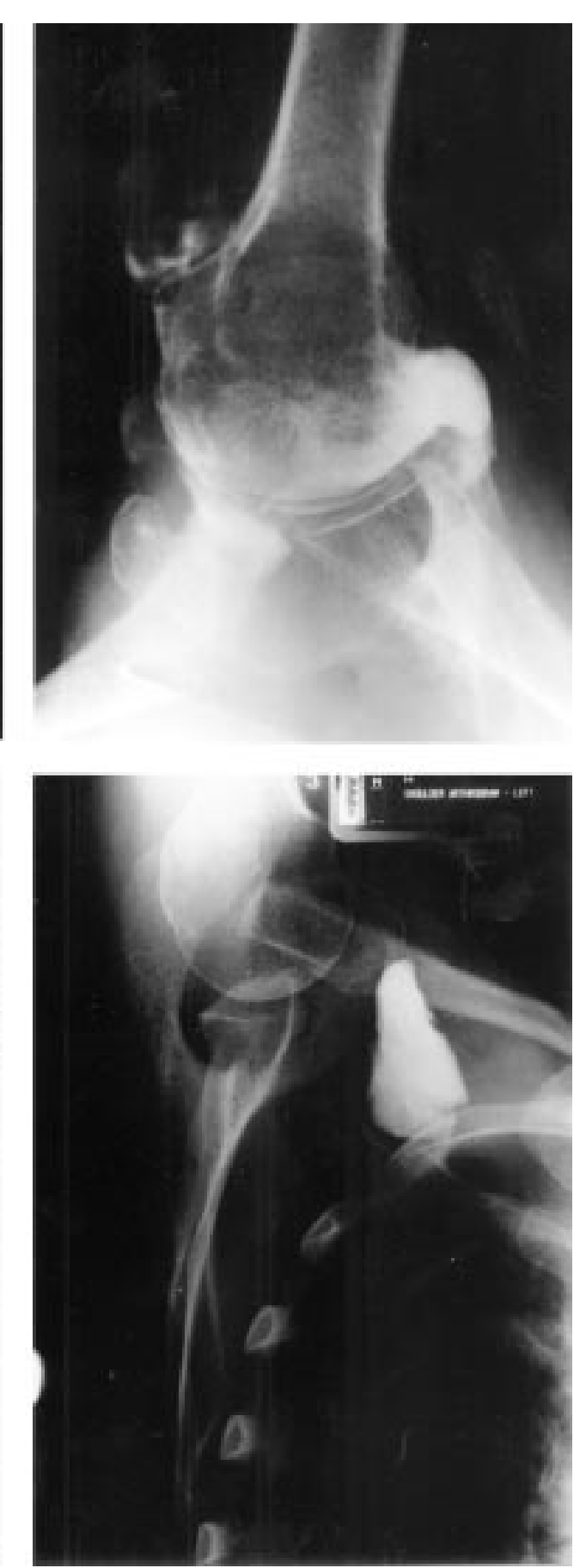

radiographic findings. At follow up, patients were questioned about the presence and duration of any side effects they attributed to the injection. The patients' assessment of both the maximum and the current global effectiveness of the procedure was also recorded using a five point qualitative rating scale ( -1 to 3$)$, namely : -1 , worse than at baseline; 0 , no benefit; +1 , small benefit; +2 , moderate benefit, or +3 , great benefit. Finally the patients were asked if they were willing to have the procedure repeated if necessary.

Having checked for normality, the mean and standard deviation were calculated at baseline, 
Table 1 Demographic details

\begin{tabular}{lcc}
\hline Total number & 37 & \\
Male & 9 & $(24 \%)$ \\
Female & 28 & $(76 \%)$ \\
Age (years) & & \\
$\quad$ Mean & 58.6 \\
$\quad$ Range & $22-75$ \\
Duration of symptoms (months) & \\
$\quad$ Mean & 8.9 \\
Range & $2-24$ \\
\hline
\end{tabular}

for each of the seven measured variables: pain, stiffness, loss of function, flexion, abduction, and internal and external rotation. Ninety five per cent confidence intervals (CI) for the change in each variable from baseline were then calculated for the accurate and inaccurate subgroups, with significant differences being determined using a two tailed Student $t$ test. The $95 \%$ CI for the difference in the change from baseline between the accurately and inaccurately injected groups, for each of the seven measured variables, was also calculated.

Comparison between the accurately and inaccurately injected groups for the two non-parametric five point rating scales (current and maximum benefit) was performed using a Mann-Whitney rank sum test.

\section{Results}

Thirty seven patients were enrolled in the study; one patient received injections to both shoulders, resulting in a total of 38 study procedures. The demographic details of the study population are shown in table 1 . The background rheumatological diagnosis included six patients with rheumatoid arthritis, 10 with generalised osteoarthritis, and one with ankylosing spondylitis. Twenty one patients had no underlying rheumatological diagnosis. Post-study analysis showed that there was no statistical difference in the underlying rheumatological diagnoses between the accurate and inaccurate subgroups (data not shown).

The pre-injection diagnoses of the affected shoulders and the type of injection used is

Table 2 Number of accurately (A) and inaccurately (I) injected joints according to the underlying shoulder diagnosis and injection type

\begin{tabular}{|c|c|c|c|c|c|c|}
\hline & \multicolumn{2}{|c|}{ Glenohumeral } & \multicolumn{2}{|c|}{ Subacromial } & \multicolumn{2}{|l|}{ Total } \\
\hline & $A$ & $I$ & $A$ & $I$ & $A$ & $I$ \\
\hline Rotator cuff tendinitis & 3 & 0 & 4 & 10 & 7 & 10 \\
\hline Adhesive capsulitis & 5 & 11 & 0 & 0 & 5 & 11 \\
\hline Acute synovitis & 2 & 3 & 0 & 0 & 2 & 3 \\
\hline Total (\%) & $10(42)$ & $14(58)$ & $4(29)$ & $10(71)$ & $14(37)$ & $24(63)$ \\
\hline
\end{tabular}

Table 3 Mean (SD) baseline values and mean (95\% confidence interval) values for the improvement after injection in the accurately and inaccurately injected subgroups

\begin{tabular}{|c|c|c|c|c|}
\hline & \multicolumn{2}{|l|}{ Baseline } & \multicolumn{2}{|l|}{ Change from baseline } \\
\hline & Accurate & Inaccurate & Accurate & Inaccurate \\
\hline $\mathrm{P}$ & $6.5(1.2)$ & $6.8(1.5)$ & 3.3 (2.1 to 4.5$)$ & $1.7(0.7$ to 2.7$)$ \\
\hline$S$ & $5.6(2.1)$ & $5.3(2.5)$ & $2.9 \quad(1.9$ to 3.9$)$ & $0.9(0.1$ to 1.7$)$ \\
\hline LOF & $6.1(2.1)$ & $5.6(2.4)$ & 2.8 (1.9 to 3.7$)$ & $0.4(-0.6$ to 1.4$)$ \\
\hline FLX & $129 \quad(42)$ & $120 \quad(50)$ & $20 \quad(10$ to 30$)$ & $8(2$ to 14$)$ \\
\hline $\mathrm{ABD}$ & $126 \quad(56)$ & 119 (44) & $17 \quad(7$ to 27$)$ & $2 \quad(-4.4$ to 8.4$)$ \\
\hline IR & 59 (18) & $62 \quad(18)$ & $0.78(1.3$ to 12.7$)$ & $2 \quad(-3.2$ to 7.2$)$ \\
\hline ER & $56 \quad(19)$ & $52 \quad(27)$ & $11 \quad(5.2$ to 16.8$)$ & $3 \quad(-1.8$ to 7.8$)$ \\
\hline
\end{tabular}

P, power; S, stiffness; LOF, loss of function; FLX, flexion; ABD, abduction; IR, internal rotation; ER, external rotation.
Table 4 95\% Confidence intervals for the difference in change from baseline between the accurately and inaccurately injected subgroups

\begin{tabular}{lc}
\hline Power & -0.04 to 3.24 \\
Stiffness & 0.67 to $3.30^{\star}$ \\
Loss of function & 0.98 to $3.82^{\star}$ \\
Flexion & 0.32 to $33.68^{\star}$ \\
Abduction & 3.03 to $26.97^{\star}$ \\
Internal rotation & -3.21 to 13.21 \\
External rotation & -22.38 to 38.38
\end{tabular}

$\star \mathrm{P}<0.05$.

shown in table 2 . Three of the 17 patients with rotator cuff syndrome were injected using an anterior approach, as the area of maximum tenderness was situated over the anterior joint line. Fourteen of the 38 procedures were judged to be accurately placed, giving an overall success rate of approximately $37 \%$ : four of the 14 attempted subacromial injections were accurately injected (29\%), as were 10 of the 24 attempted glenohumeral injections (42\%).

The mean and standard deviation values at baseline, as well as the mean and $95 \%$ CI for the change from baseline, for both the accurate and inaccurate subgroups are shown on table 3. There was no significant difference between the two subgroups at baseline. Table 4 shows the comparison of $95 \%$ CI values for the difference in the change from baseline between the accurately and inaccurately injected subgroups. Significant differences between the two subgroups were present, with regard to stiffness, loss of function, and for the change in flexion and abduction.

Similarly the perceived maximum benefit was significantly better in the accurately injected subgroup (table 5). Four of the 14 accurately injected patients $(28.6 \%)$ reported that they had found the procedure to be of "great benefit", while only one of the 24 patients in whom the injection was inaccurate (7\%) reported this level of maximum benefit. At the time of follow up, the current benefit approached, though did not reach, significance, with a $P$ value of 0.07 .

Ten patients reported some degree of discomfort which they attributed to the injection. In only three cases did this persist beyond the day of the injection. All such symptoms had resolved by the time of follow up and none required the patient to seek medical attention. Only three of the 37 patients expressed reluctance at follow up to have the procedure repeated if required.

\section{Discussion}

Although they have been in general clinical use for over 40 years, the role of steroid injections in the management of localised shoulder complaints remains uncertain. Clinical experience

Table 5 Comparison of the median (range) for the perceived maximum benefit after injection $(-1$ to +3$)$ and the current level of benefit $(-1$ to +3$)$ between the accurately and inaccurately injected subgroups

\begin{tabular}{llll}
\hline & Accurate & Inaccurate & P value \\
\hline $\begin{array}{c}\text { Maximum } \\
\text { benefit }\end{array}$ & $2(1$ to 3$)$ & $1(0$ to 3$)$ & 0.034 \\
$\begin{array}{c}\text { Current } \\
\text { benefit }\end{array}$ & $2(0$ to 3$)$ & $1(0$ to 3$)$ & 0.07 \\
\hline
\end{tabular}


suggests that, while some patients respond dramatically to local steroid placement, others respond at best poorly and some not at all. Controlled trials of the efficacy of steroid placement have been relatively few and have provided conflicting results. Most have had different methodologies, thus making comparison between studies difficult, while many have had relatively small numbers in the individual treatment groups, thus limiting their statistical power.

Several prospective studies comparing steroid placement with other interventionsincluding the local injection of lignocaine, used in many studies as a relative placebo intervention - failed to demonstrate any lasting benefit for the steroid injected group. ${ }^{6-11}$ Other studies, however, have found significant improvements in the steroid injected patients compared to controls. ${ }^{12-14}$

None of these studies attempted to assess the accuracy with which the steroids were injected. It is therefore possible that the differences in outcome may have resulted as much from variability in the accuracy of steroid placement as from the efficacy of the steroid treatment itself.

In a study by Weiss and Ting, ${ }^{16}$ the accuracy of placement was ensured by injecting the shoulder at the time of arthrography, using the actual cannula through which the contrast material had been introduced into the joint. Of the 18 patients with adhesive capsulitis treated in this fashion $16(88.8 \%)$ had a successful outcome.

Of the various joints commonly injected, the glenohumeral joint is one of the more difficult, especially when it has undergone significant arthritic deformity. This difficulty in entering an arthritic glenohumeral joint is well appreciated by radiologists experienced in performing shoulder arthrography. ${ }^{17}$ In his study on local steroid placement Richardson commented that success in the accurate injection of the glenohumeral joint was achieved only "inconstantly". ${ }^{13}$ Weiss and Ting failed to enter the glenohumeral joint successfully in eight of 66 attempted arthrograms despite having the benefit of fluoroscopic guidance. ${ }^{16}$ In a study of 108 attempted intra-articular injections at various sites, Jones et al found only 56 which were definitely intra-articular. ${ }^{18}$ Of the 20 attempted glenohumeral injections in Jones's study only two were judged to be definitely accurate.

The patients in our study represent the spectrum of patients attending a busy rheumatology outpatient clinic, as well as the range of shoulder syndromes which are treated with localised steroid injections. Essential to the accuracy with which the position of the injection could be judged was that the injections were performed in the radiology suite itself, the patient being radiographed immediately afterwards in two standardised planes. Thus we avoided the difficulty experienced in Jones's study where the position of 21 of the 108 injections could not be judged as no contrast material was visible. ${ }^{18}$
The overall success rate for glenohumeral injection in our study, using a standardised anterior approach, was only $42 \%$. Whether a posterior approach might result in greater accuracy and superior results remains unestablished.

As the patients' symptoms were present for a minimum of two months and a mean duration of eight months it seems reasonable to attribute the changes seen at follow up to the steroid injection rather that to the natural history of the shoulder complaint. While both the accurately placed and the inaccurately placed groups improved with the injection, the improvements were significantly better for the former. Though periarticular placement may allow sufficient steroid to diffuse into an adjacent joint and so achieve a partial response, a far greater concentration of drug and much less dispersal away from the desired target would be expected if the injected material is successfully placed within the confines of either the intra-articular space or a bursa.

As expected, the procedures were in general well tolerated. At the follow up assessment no patient had persistent symptoms attributed to the injection itself and the vast majority of patients were happy to have the procedure repeated if necessary.

In summary, we report the first prospective study examining the effect of the accuracy of steroid placement on the clinical outcome in patients with shoulder symptoms. Our results show that while local steroid injections are in general well tolerated, they are frequently inaccurate. In many cases the steroids are not injected into the desired target. It is not surprising, given the difficulty that may be experienced in entering an arthritic shoulder joint even with the benefit of fluoroscopy, that there should be a high failure rate in attempting blind joint entry. However, the fact that in both our study and that of Jones et al ${ }^{18}$ over half the attempted shoulder injections were inaccurately placed is nonetheless disappointing. It perhaps calls into question the methods which we use to teach these techniques and the manner in which we employ them in our everyday clinical practice. Furthermore, in patients who fail to respond optimally to blind shoulder injections, consideration should be given to the use of some form of imaging in an attempt to improve the accuracy of steroid placement and the results that may be achieved with this cheap and potentially effective treatment.

1 Dacre J, Beeney N, Scott D. Injections and physiotherapy for the painful stiff shoulder. Ann Rheum Dis 1989; 48:322-5.

2 Jacobs I, Barton M, Wallace W, Ferrousis J, Dunn N, Bossingham D. Intra-articular distension and steroids in the management of capsulitis of the shoulder. BMJ 1991; 302:1498-501.

3 Sparling M, Malleson P, Wood B, Petty R. Radiographic follow up of joints injected with triamcinolone hexacetonide for the management of childhood arthritis. Arthritis Rheum 1990;33:821-6.

4 Von Essen R, Savolainen H. Bacterial infection following intraarticular injection. Scand J Rheumatol 1989;18:7-12.

5 Hollander J. Arthrocentesis and intrasynovial therapy. In: McCarty D, ed. Arthritis and allied conditions, 9th ed. Philadelphia: Lea \& Febiger, 1979:402-14. 
6 Lee P, Lee M, Haq A, Longton E, Wright V. Periarthritis of the shoulder. Ann Rheum Dis 1974;33:116-9.

7 Rizk T, Pinals R, Talaiver A. Corticosteroid injections in adhesive capsulitis: investigation of their value and site. Arch Phys Med Rehabil 1991;72:20-2.

8 White R, Paull D, Fleming K. Rotator cuff tendonitis: comparison of subacromial injection of a long acting corticosteroid versus oral indomethacin therapy. J Rheumatol 1986;13:608-13.

9 Berry H, Fernandes L, Bloom B, Clark R, Hamilton E. Clinical study comparing acupuncture, physiotherapy, injection and oral anti-inflamatory therapy in shouldercuff lesions. Curr Med Res Opin 1980;7:121-6.

10 Bulgen D, Binder B, Hazleman B, Dutton J, Roberts S. Frozen shoulder: perspective clinical study with an evaluation of three treatment regimens. Ann Rheum Dis 1984; 43:361-4.

11 Vecchio P, Hazleman B, King R. A double-blind trial comparing subacromial methylprednisolone and lignocaine in acute rotator cuff tendonitis. $\mathrm{Br}$ J Rheumatol 1993.

12 Petri M, Dobrow R, Neiman R, Whiting-O'Keefe Q, Seaman W. Randomized, double-blind, placebocontrolled study of the treatment of the painful shoulder. Arthritis Rheum 1987;30:1040-5.

13 Richardson A. The painful shoulder. Proc R Soc Med 1975; 68:731-6.

14 Adebajo A, Nash P, Hazleman B. A prospective double blind dummy placebo controlled study comparing triamcilone hexacetonide injection with oral Diclofenac $50 \mathrm{mg}$ TDS in patients with rotator cuff tendonitis. J Rheumatol 1990; 17:1207-10.

15 Dixon A, Graber J. Local injection therapy in rheumatic disease, 2nd ed. Basle: Eular publications, 1983:74-83.

16 Weiss J, Ting $M$. Arthrography-assisted intra-articular injection of steroids in treatment of adhesive capsulitis. Arch Phys Med Rehabil 1978;59:285-7.

17 Stoker D. Intra-articular injections in capsulitis [letter]. BMJ 1991;303:123.

18 Jones A, Regan M, Ledingham J, Pattrick M, Manhire A, Doherty M. Importance of placement of intra-articular steroid injections. BMJ 1993;307:1329-30.

$A$ representation from the anterior aspect of the bones of the human body articulated together.

The Anatomical Drawings of Andreas Vesalius, 1543. 